\title{
Study of Polysulfone and Polyacrylic Acid (PSF/PAA) Membranes Morphology by Kinetic Method and Scanning Electronic Microscopy
}

\section{Chamekh Mbareck ${ }^{1 *}$ and Quang Trong Nguyen ${ }^{2}$}

${ }^{1}$ Université des Sciences, de Technologie et de Médecine; Faculté des Sciences et Techniques, B.P. 5026, Nouakchott, Mauritanie ${ }^{2}$ P.B.S. UMR 6270 CNRS - Université de Rouen, 76821 Mont-Saint-Aignan, France

\begin{abstract}
This work focuses on the study of the morphology forming of Polysulfone (PSf) and polyacrylic acid (PAA) membranes which are prepared by mixing both of the polymers in DMF and the obtained blend is precipitated in water (non-solvent). The precipitation kinetic and the effects of polysulfone concentration, drying-time in free-air and proportions of both polymers are investigated.

Based on the SEM technique, the kinetic and viscosity measurements, and the visual observations, this study brings to the fore the different steps of the morphology forming of PSf/PAA membranes: formation of finger-like structures, sponge-like-structures, inner pores, superficial pores and the craters. Appearance of these structures is governed by the exchange process, between casting polymer-solution and the precipitation-bath (coagulation), which is controlled by operating conditions. This work constitutes a great tool in understanding the mechanisms of the morphology forming of PSf/PAA membranes and to improve their performances: preparation of tailored membrane.
\end{abstract}

Keywords: Polysulfone; Poly (acrylic acid); Membrane morphology; SEM; Blends; Macrovoids

\section{Introduction}

Polysulfone is a hydrophobic polymer with excellent chemical and mechanical properties. However, the hydrophobic behaviour prevents its use in several areas such as in water treatment. In order to overcome this drawback, some researchers have proposed the incorporation of hydrophilic groups, to increase the polymer hydrophilicity which improves the membrane water- permeability and reduces membranefouling $[1,2]$.

In literature, the synthesis of carboxylated polysulfone membranes was realized through different ways. Membranes were prepared by (i) substitution with functionalized groups, (ii) by polymer graft modification or (iii) by surface modification of prefabricated membranes [2-9].

In a previous, work we proposed the preparation of carboxylated polysulfone membranes by mixing PSf and PAA in dimethy formamide (DMF) solvent and, the obtained blend, was precipitated in waterbath (non-solvent) [10]. Incorporation of carboxylic groups in PSf matrix imparted the obtained membrane high ionic-exchange and complexation capacities. It also increased the membrane hydrophilicity which enhanced the resistance to organic fouling and made membrane suitable for many applications. As a result, it was found that, the membrane properties depended on: (i) uniform distribution of PAA chains in the network formed by the PSf matrix, (ii) rate of immobilization of PAA, and (iii) morphology of the membrane. The PSf/ PAA membrane has shown good performances for the ultrafiltration of different heavy metal solutions and dye molecules [10,11]. Here, our interest will be focused on the PSf/PAA membrane morphology in order to learn much about the mechanisms of the morphology forming and to know how to improve the membrane performances in view to prepare tailored membranes.

SEM technique, visual observation, precipitation speed, viscosity measurement are used to characterize the morphology of the PSf/PAA membranes.

\section{Experiment}

\section{Polymer properties}

Polysulfone (PSf) and poly (acrylic acid) (PAA) were supplied by Aldrich and dimethyl formamide (DMF) by Prolabo. All polymers and chemical products were used as supplied, without any further purification. Molecular weights of PAA and PSf were 450000 and 26 $000 \mathrm{~g} / \mathrm{mol}$, respectively.

\section{Membrane preparation}

Polysulfone (PSf) and Poly (acrylic acid) (PAA) were dissolved, separately, in dimethyl formamide (DMF) in a glass reactor equipped with a mechanical stirrer and thermostated at $90^{\circ} \mathrm{C}$ for over 3 hours. The PSf concentration was 17 (or 19) wt. \% and that of PAA was 5 wt. \%. Afterwards, both solutions were mixed together in known proportions, stirred for $30 \mathrm{~min}$ and de-bubbled. Such de-bubbled casting-solution was casted on a glass plate with a lab-made Gardner knife, dried in freeair for a known time (generally 20 seconds) and finally immersed in a coagulation-bath containing a sufficient volume of MilliQ water (18.2 $\mathrm{M} \Omega \mathrm{cm})$ at $18^{\circ} \mathrm{C}$. Membranes were thoroughly washed with water, and stored in a dilute sodium azide solution till their use.

\section{Kinetic measurement}

Five polymer-solutions with the same mass $(2 \mathrm{~g})$ and different composition (PSf/PAA 100/0, 96/04, 92/08, 89/11 and 83/17) were poured on a glass plate to get exactly a same surface area. Afterwards,

*Corresponding author: Chamekh MBareck, Université des Sciences, de Technologie et de Médecine; Faculté des Sciences et Techniques, B.P. 5026 Nouakchott, Mauritanie, Tel: +222 52513 82; E-mail: chamec1@yahoo.fr

Received August 28, 2014; Accepted October 26, 2014; Published October 30 2014

Citation: Mbareck C, Nguyen QT (2014) Study of Polysulfone and Polyacrylic Acid (PSF/PAA) Membranes Morphology by Kinetic Method and Scanning Electronic Microscopy. J Membra Sci Technol 4: 132. doi:10.4172/2155-9589.1000132

Copyright: (c) 2014 Mbareck C, et al. This is an open-access article distributed under the terms of the Creative Commons Attribution License, which permits unrestricted use, distribution, and reproduction in any medium, provided the original author and source are credited. 
Citation: Mbareck C, Nguyen QT (2014) Study of Polysulfone and Polyacrylic Acid (PSF/PAA) Membranes Morphology by Kinetic Method and Scanning Electronic Microscopy. J Membra Sci Technol 5: 132. doi:10.4172/2155-9589.1000132

Page 2 of 7

polymer casting-solution is immersed in equal volume of water coagulation-bath (2 liters).

Kinetic measurements were used to determine the time and the speed of PSf/PAA precipitation. The time of precipitation corresponds to the period separating the moment of immersion of casting-solution in the water-bath and the moment at which the formed membrane peels spontaneously off from the glass plate. The speed of precipitation was calculated according to the following formula: $S_{p}=\mathbf{m} / \mathbf{t}_{\mathbf{p}}$

Where, $S_{p}$ is the precipitation speed of the polymer solution (in $\mathrm{gr} / \mathrm{sec}$ ); $\mathrm{m}$ : is the mass of casting-solution (in gram); $\mathrm{t}_{\mathrm{p}}$ : is the time of precipitation (in second).

\section{Viscosity measurement}

Rheological experiments were carried out using a controlled shear stress (advanced rheometer: AR 2000 from TA instruments). A double

\begin{tabular}{|c|c|c|c|c|c|}
\hline PAA\% & 0 & 0.04 & 0.08 & 0.11 & 0.17 \\
\hline $\mathrm{S}_{\mathrm{p}}$ (gr./Sec) & 0.066 & 0.055 & 0.029 & 0.027 & 0.025 \\
\hline
\end{tabular}

Table 1: Precipitation speed of polymer casting-dopes as function of PAA percentage.

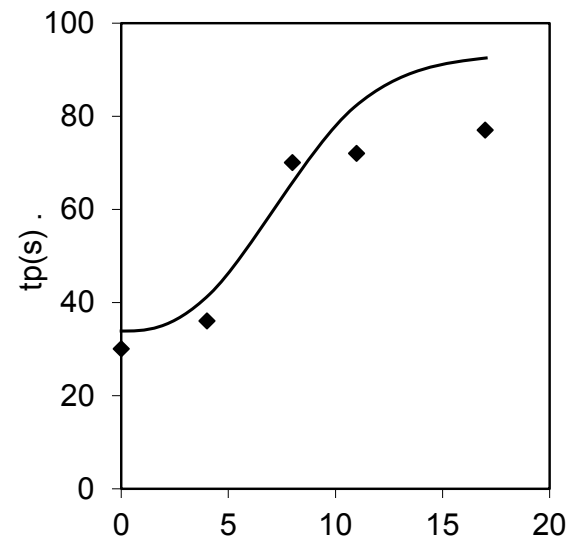

$\%$ PAA

Figure 1: Precipitation time for PSf/PAA blends at different PAA fraction.
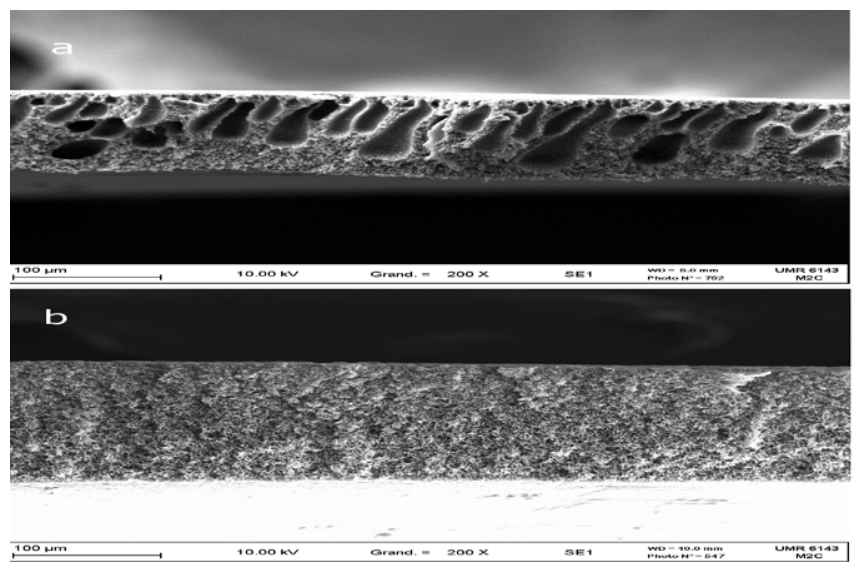

Figure 2: SEM micrographs of PSf membranes for (a) PSf/DMF 17/83 and (b) PSf/DMF 19/81. gap concentric cylinder device was used. Measurements were done at $25 \pm 0.1^{\circ} \mathrm{C}$ with the shear rates ranging from 0.1 to $660 \mathrm{~s}^{-1}$ and shear stress from 0.01 to $622 \mathrm{~Pa}$.

\section{Scanning Electronic Microscopy}

Membrane surfaces and sections were analyzed by a Zeiss EVO 40 EP microscope with the Secondary Electron Backscattered Detector (CE-BSD). Samples were frozen in liquid nitrogen at $-190^{\circ} \mathrm{C}$, fractured and vacuum coated with a thin gold film. The SEM observations were carried out at various magnifications while the electron beam energy was fixed at $10 \mathrm{keV}$.

\section{Results and Discussion}

\section{Kinetic measurement}

Kinetic measurements aim at characterizing the behaviour of PSf/ PAA membranes during precipitation step. Therefore, precipitations time and speed were determined. It is interesting to note that precipitation time may be also regarded as time of total exchange of solvent and non-solvent between casting-solution and precipitationbath.

As shown in Table 1, the high precipitation speed of PSf solutionfilm, compared with those of PSf/PAA solution-films, is the consequence of larger difference in the solubility parameter values of PSf $\left(12.9 \mathrm{cal}^{1 / 2} /\right.$ $\left.\mathrm{cm}^{3 / 2}\right)$ and water $\left(23.4 \mathrm{cal}^{1 / 2} / \mathrm{cm}^{3 / 2}\right)$ [12]. The addition of PAA $\left(14.6 \mathrm{cal}^{1 / 2} /\right.$ $\mathrm{cm}^{3 / 2}$ ) on the PSf solution - to form a blend - decreases the difference in solubility between polymer-solution and water; and increases the precipitation time of PSf/PAA blends as shown in Figure 1 [13].

It's well known that PAA is a hydrophilic polymer which can develop strong interactions with water. Then, the increase of PAA content in the casting-solution reduces chemical driving forces of exchange with nonsolvent and such a thing delays the precipitation of the casting-solution $[14,15]$.

As a result, the precipitation of PSf/PAA blends depends on the rate of the solvent expulsion from casting-solutions. This expulsion is governed by the difference in the solubility parameter or the affinity (interactions) between polymer and coagulation-bath.

\section{Effect of the PSf concentration in the casting- solution}

Figure $2 \mathrm{a}$ and $\mathrm{b}$ presents the SEM micrographs of PSf membranes obtained from the casting-solution containing, respectively, $17 \%$ and 19 wt. \% of PSf in DMF. These micrographs show that with the increase in the PSf concentration, the membrane morphology changes from finger-like structures to sponge- like structures. Some researches assign the appearance of finger-like structures (macrovoids) to instantaneous precipitation of PSf-film [15-17]. Here, suppression of macrovoids, within membrane with higher percent (19 wt \% PSf), is mainly due to the increase of the viscosity of casting-solution as both of the castingsolutions have practically the same precipitation time $[15,18]$.

At higher polymer content, growth of the nucleus of poor-polymer phase is disrupted by the high viscosity of the liquid medium; the vitrification of the rich-polymer matrix surrounding the nucleus would occur more easily. However, the macrovoid suppression is accompanied by a change in the porosity, pore size and pore distribution in the membrane [19-21].

\section{Effect of drying time in free air (pre-coagulation) $\left(\mathrm{t}_{\mathrm{d}}\right)$}

Three PSf/PAA 96/04 membranes are used to study the effect of drying time on the membrane morphology. Two samples are, 
Citation: Mbareck C, Nguyen QT (2014) Study of Polysulfone and Polyacrylic Acid (PSF/PAA) Membranes Morphology by Kinetic Method and Scanning Electronic Microscopy. J Membra Sci Technol 5: 132. doi:10.4172/2155-9589.1000132
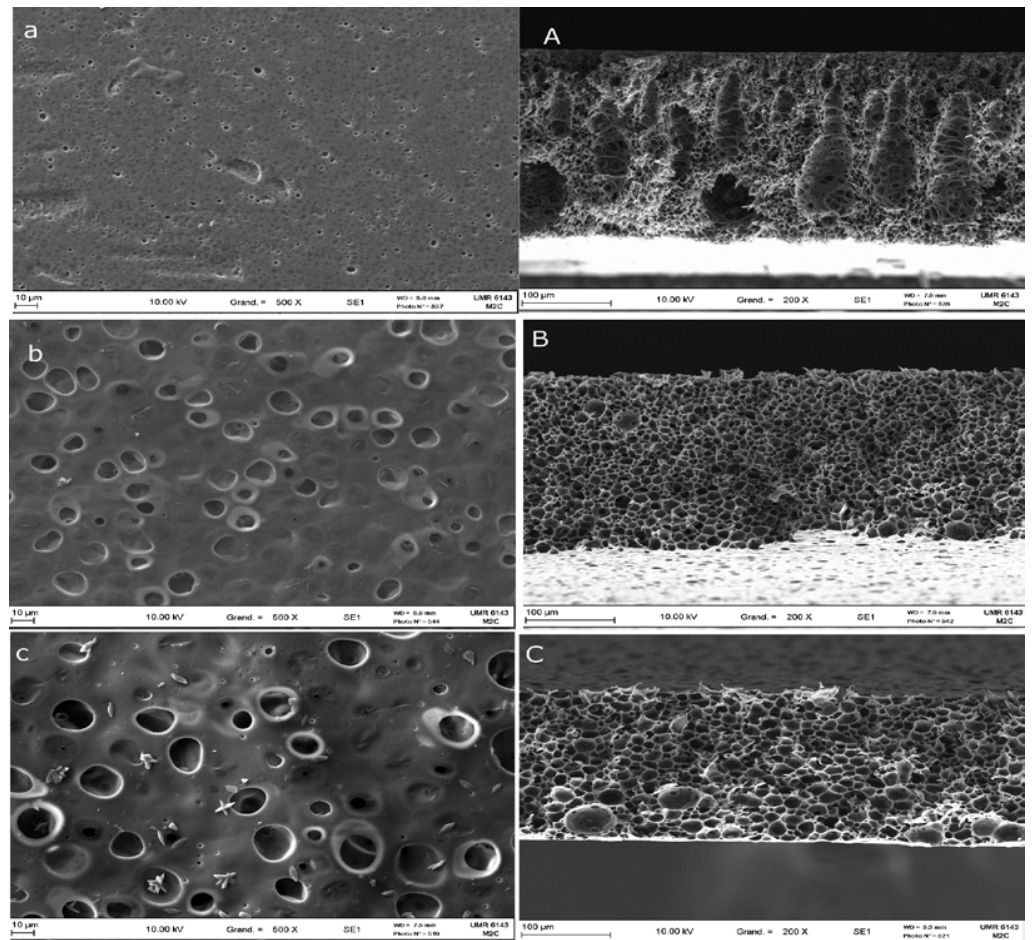

Figure 3: Surface and section SEM micrographs of PSf/PAA 96/04 at different precoagulation drying time: (a, A) 10 sec, (b, B) 300 sec and (c, C) $+\infty$, respectively. The magnification of surface images is $500 \mathrm{X}$ whereas that of section images was 200X. The concentration of initial PSf/DMF solution was $19 \%$ (wt).

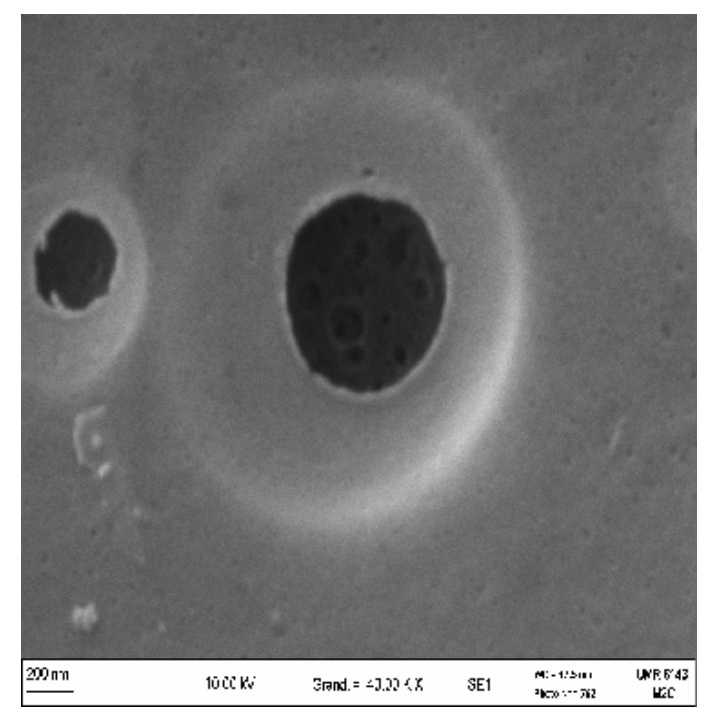

Figure 4: SEM image of PSf/PAA 92/08 membrane with different levels of pore structure $(40000 \mathrm{X}, 10 \mathrm{KV})$.

respectively, dried during 10 and 300 seconds before immersing in water-bath whereas the third is completely dried in free air (water vapour $40 \%$ ).

This experiment shows that the size of superficial pore increases with the increase in drying time (Figure 3), and macrovoids in the section are completely suppressed for $t_{d}$ higher than 300 seconds (Figure 3C). The size of superficial pores increases from $100 \mathrm{~nm}$ at a short time to $10 \mu \mathrm{m}$ at a long drying time.
In fact, it is difficult to give a single value to the pore size as the majority is composed of different porous structures as shown in Figure 4. The SEM image of membrane surface shows that each superficial pore contains smaller inner-pores at different levels. For instance, the average size of the superficial pore in the PSf/PAA 92/08 membrane was ca. $680 \mathrm{~nm}$, while that of the first inner-pores level was $130 \mathrm{~nm}$ and the second inner-pores level was $25 \mathrm{~nm}$. It appears that the pore sizes in the membrane active-layer decrease progressively from the upper surface towards the inner section. Progressive decrease of these pore sizes and good performances of these membranes for ultrafiltration of heavy metal ions, published previously [11], suggest the high membrane tortuosity from one face to another.

The exposure of PSf/PAA/DMF film in free-air provokes an exchange between the solvent in casting-film (DMF, b.p. $152.8^{\circ} \mathrm{C}$ ) and the atmospheric water vapour (at $40 \%$ relative humidity) [22]. Due to the low solvent volatility and low water content in air, the exchange rate of solvent and nonsolvent (water) is lower in free-air than in water-bath.

A short drying time (a rapid quenching) of the casting liquidfilm in the coagulation bath induces a rapid film vitrification (phase separation) due to further solvent outflow. This phenomenon provokes high growth of rich-polymer phase in the sub-layer and growth of poor-polymer phase in the bottom layer. These circumstances favor the formation of finger-like structure forms. For long drying time, the exchange of solvent and non-solvent is slow, as well as, the solutionfilm vitrification. This behavior leads to a more homogeneous structure (more sponge- like structure).

The precipitation of casting-solution in free-air is very slow in comparison with the precipitation in water-bath which is fast. In fact, the high boiling-point of solvent and low inflow rate of water-vapour 
Citation: Mbareck C, Nguyen QT (2014) Study of Polysulfone and Polyacrylic Acid (PSF/PAA) Membranes Morphology by Kinetic Method and Scanning Electronic Microscopy. J Membra Sci Technol 5: 132. doi:10.4172/2155-9589.1000132

Page 4 of 7
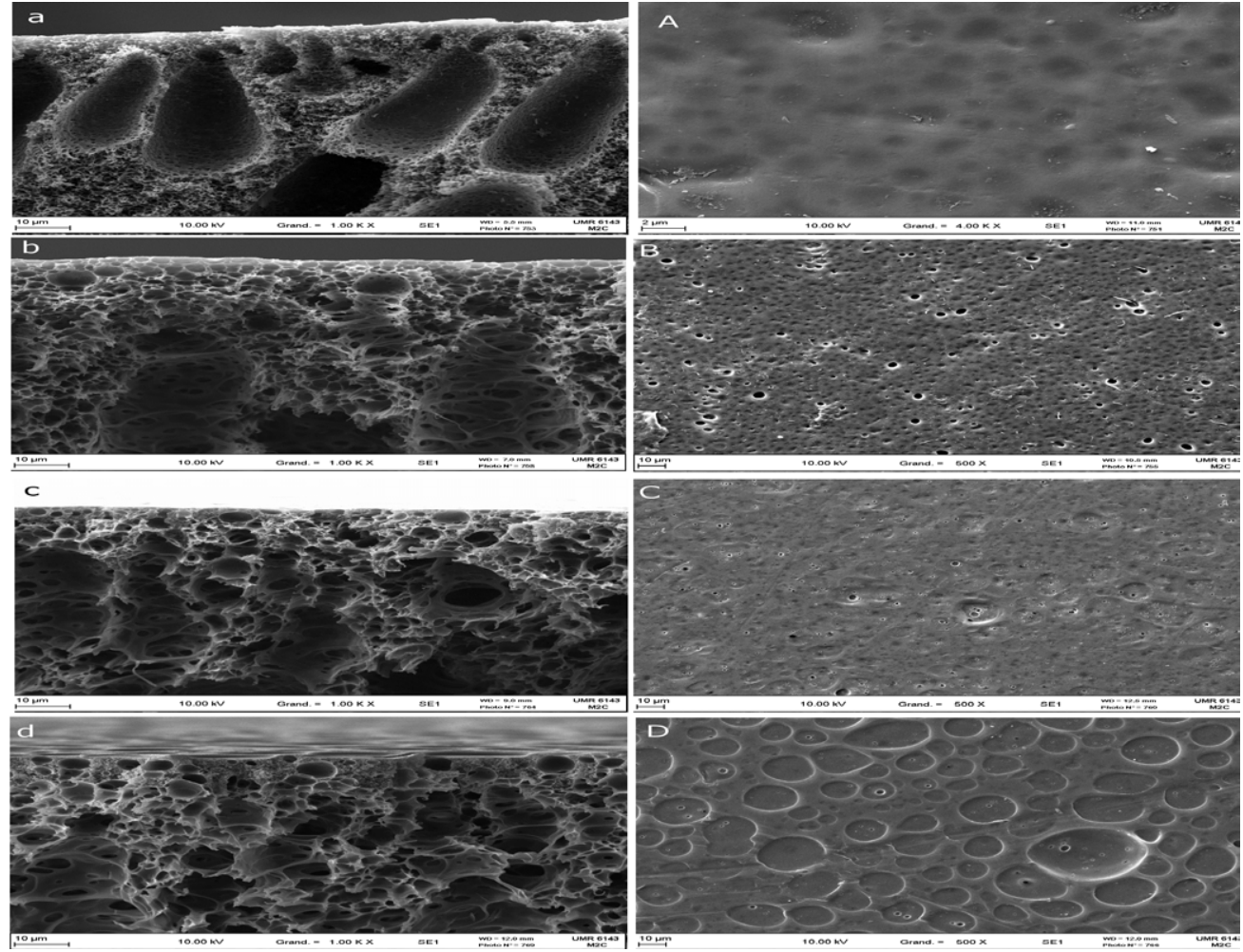

Figure 5: Cross- section SEM micrographs of PSf/PAA membranes (a) PSf/PAA 100/0, (b) 96/04, (c) 92/08 and (d) 89/11. The concentration of initial PSf/DMF solution was $17 \%(\mathrm{wt})$

in casting-solution reduced sensibly the exchange driving forces between casting-solution and water. Thus, the vitrification of solutionfilm progresses slowly leading to homogeneous matrix with inner and superficial pores.

These results show again the role of the driving forces arising from solvent and non-solvent exchange: rapid exchange of solvent and nonsolvent favors the formation of finger-like structures, whereas the slow exchange enhances the formation of sponge-like structures.

\section{Effect of the blend composition}

Figure 5 shows the cross- section and surface morphologies of PSf/ PAA $100 \%, 96 / 04,92 / 08$ and 89/11, respectively. The SEM micrographs show that the morphology of PSf $100 \%$ is mainly composed of fingerlike structures which progressively disappear in favor to sponge-like structures with the increase in PAA content.

The progressive suppression of finger-like structure is in agreement with the previous results (section 3.1). The addition of PAA increases the intermolecular interactions between casting-solution and the nonsolvent (water). Such a thing delays the precipitation of casting-solution and enhances the homogenous structures forming. These results are in agreement with those of $\mathrm{Wu}$ et al. [23] who suggest that weak interactions between polyvinylidene fluoride and poly (ethersulfone) chains tend to create a large distance between the polymers chains which induces the formation of macrovoid-structures.

\section{Visual observation of casting-solution precipitation}

Visual observation of the precipitation of PSf/PAA/DMF castingsolution shows a strong film-contraction and thin turbulence-liquid streams which escape rapidly from the top-face of the nascentmembrane. These liquid-streams appear at numerous superficial points. Afterwards, the film peels off from the glass plate pushed by a large amount of solvent which emerges from the membrane bottomface. This phenomenon strength decreases with the increase of PAA percentage.

The simple analysis of this phenomenon leads to admit that, the immersion of PSf/PDF casting-solution in non-solvent-bath creates strong repulsive forces between PSf chains (hydrophobic) and water molecules (hydrophilic). These forces provoke the rapid retraction of polymers' chains and the formation of rich-polymer domains and rich-liquid domains. The progressive retraction of polymers' chains pushes the rich-liquid domains to escape through the polymer matrix inducing the formation of inner and superficial pores, on the one hand, and the formation finger-like structures and sponge-like structures, on the other hand. The increase of PAA percentage reduces the repulsive forces and encourages the homogeneity of membrane morphology.

\section{Interpretation of PSf/PAA morphology Mechanism}

Under the driving forces of exchange between casting-solution and non-solvent (water), large fluctuations in concentration appear at the beginning of the phase separation, leading to large stresses between the poor-polymer and the rich-polymer phases, as illustrated in Figure 6a. Transient gel-clusters would be formed due to the dynamic asymmetry in stresses between the system components. Afterwards, the clusters aggregate into large gel domains [24]. Chemical potential changes induce an osmotic pressure which causes contraction of the elastic gel domains and liquid expulsion as illustrated in Figure $6 \mathrm{~b}$. 

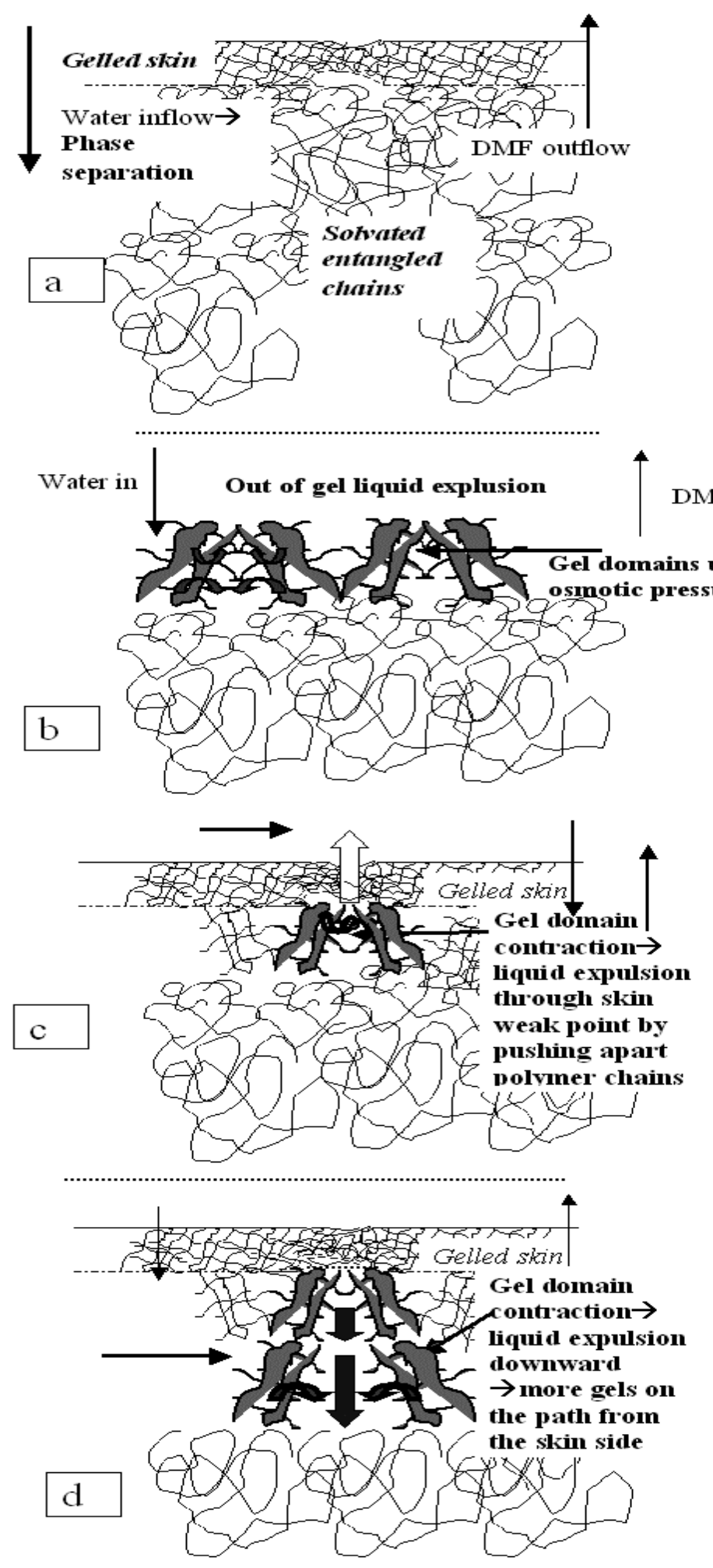

Figure 6: The mechanism of the formation of macrovoids structure and surface craters. Evolution in time of the structure of the solution of polymer film cast on a glass plate:

a) in the first step of polymer gelling into a surface gel skin, just after immersion in the water coagulation bath ;

b) seconds later, in the next step of gel domain formation in the sub - gel skin layer ;

c) in the step of formation of a surface crater by solvent ejection under gel collapse pressure through weak points in the gel skin, followed by a self- sealing of gel skin at the break point ; d) in the step of formation of a macrovoid by water- rich solvent flow towards the bottom face under gel collapse pressure, and subsequent preferential phase separation and gel domain formation on the flow path. 


\begin{tabular}{|l|c|c|c|c|c|}
\hline PAA $\%$ & 0 & 0.04 & 0.08 & 0.11 & 0.15 \\
\hline$\eta_{0}(\mathrm{~Pa} \mathrm{~S})$ & 0.6 & 1.3 & 3.6 & 5.5 & 14.6 \\
\hline Average superficial pore size $(\mu \mathrm{m})$ & 0 & 2.36 & 0.49 & 0.42 & -- \\
\hline
\end{tabular}

\begin{tabular}{|l|l|l|l|l|}
\hline Average superficial pore size $(\mu \mathrm{m})$ & 0 & 2.36 & 0.49 & 0.42 \\
\hline
\end{tabular} superficial pore size of PSf/PAA membranes as a function of PAA percentage.

Gel-collapse is physically described by Tanaka et al. [24] who propose that the water penetration induces stresses (osmotic stresses) throughout the different parts of gel structures and the liquid squeezing out of gel. As a result, rich polymer-domains and rich liquid-domains are formed across the gel. If the gel domain is closer to the surface, it will be easier for the squeezed liquid to escape through the weak spots on the nascent gel layer [25]. The ejected DMF-water mixture causes turbulence streams in the water-bath that we observed in the regions surrounding the weak points on the surface, as illustrated in Figure 6c. As early as, the stress is released and the solvent escaped at the weak points on the surface of the nascent gel layer, small pores would be formed at low layer viscosity and craters at high layer viscosity. This behavior is confirmed by the decrease of average pore size and the increase of zero-shear viscosity, with the increase of PAA fraction, as shown in Table 2. The round crater observed on the upper layer is due to a local collapse of the elastic skin after the escape of the liquid from the casting-film.

When there are no breakable weak points in the gel skin, the richwater liquid would be pushed downwards to the bottom face by the collapsing gel domains. As the water transport, from the liquid-sublayer towards the bottom face, is much faster than that of molecular diffusion, the separation of the polymer-poor phase occurs faster on the path of the squeezed water-rich liquid leading to finger-like structures (macrovoids) in the membrane section. Bottom parts of these structures are generally larger than the top parts. Such morphology can also be explained on the basis of viscoelastic properties: larger the distance from the skin layer, lower the medium viscosity. Thus, the poorpolymer phase tends to expand more in the lower part of the nascent macrovoids, as illustrated in Figure 6d.

The addition of PAA increases the viscosity of polymer-solutions (as shown by Table 2) and decreases the intensity of turbulence streams observed above the nascent membrane surface. It also induces the decrease of precipitation time. All these results show that with the reduction of solvent expulsion forces or the delay of precipitation, the morphology tends to be more homogeneous and the sponge likestructures dominate as shown by Figure 5 .

Higher affinity of the casting-solution to non-solvent due to the presence of hydrophilic PAA makes the polymer-gel domains more swollen and weaker. As a result, the breaking of weak spots in the gelled skin is still possible, and the deformation of the softer-gel domains is larger at higher PAA contents, leading to larger craters on the surface.

Exploring the morphology of the membrane prepared from casting solutions of polysulfone (PSf), polyvinylpyrrolidone (PVP) and N-methylpyrrolidone (NMP), Han and Nam [26] report that a membrane with a low PVP content (5\%) shows larger macro pores than that with a high PVP content (20\%). They suggest that the introduction of a low PVP content enhanced sufficiently the demixing ability of the casting solution to provoke a rapid collapse of polymer chains and to induce the formation of macrovoids. On the contrary, at high PVP content, the increase in the viscosity of the polymer solution due to the enhanced entanglement of polymer chains hinders the solvent-nonsolvent exchange, delays the demixing of casting-solution and induces a suppression of macrovoids. These results may be interpreted by the mechanisms that we propose to explain the morphology of PSf/PAA membrane.

\section{Conclusion}

This work has elucidated many points of the morphology mechanisms forming of PSf/PAA membrane morphology. Generally, these membranes present two morphologies: sponge-like structure and finger-like structure. The appearance of these morphologies is governed by the driving forces of solvent and non-solvent exchange. These forces provoke the retraction of polymers' chains which push the solvent to escape through the polymer matrix inducing the formation of different morphology forms: inner and superficial pores, finger-like structures and sponge-like structures. The decrease of these driving forces favors the appearance of sponge-like structure whereas their increase favors the appearance of finger-like structure.

\section{References}

1. M Ulbricht, Belfort G (1996) Surface modification of ultrafiltration membranes by low temperature plasma II. Graft polymerization onto polyacrylonitrile and polysulfone. J Memb Sci 111: 193-215.

2. Mockel D, Staude E, Guiver MD (1999) Static protein adsorption, ultrafiltration behavior and cleanability of hydrophilized polysulfone membranes. J Memb Sci 158: 63-75.

3. Lu Z, Liu G, Duncan S (2005) Morphology and permeability of membranes of polysulfone-graft-poly(tert-butyl acrylate) and derivatives. J Memb Sci 250: $17-28$.

4. Fang JK, Chiu HC, Wu JY, Suen SY Preparation of polysulfone based cation - exchange membranes and their application in protein separation with a plate and frame nodule, React Funct Polym 59: 171-183.

5. Gancarz I, Pozniak G, Bryjak M, Frankiewiez A (1999) Modification of polysulfone membranes. 2. Plasma grafting and plasma polymerization of acrylic acid. Acta Polym 50: 317-326.

6. Kato K, Uchida E, Kang ET, Uyama Y, Ikada Y (2003) Polymer surface with graft chains. Prog Polym Sci 28: 209-259.

7. Nabe A, Staude E, Belfort G (1997) Surface modification of polysulfone ultrafiltration membranes and fouling by BSA solutions. J Memb Sci 133: 57-72

8. Matsumoto Y, Sudoh M, Suzuki Y, (1999) Preparation of composite UF membranes of sulfonated polysulfone coated on ceramics. J Memb Sci 158 $55-62$.

9. Béquet S, Remigy JC, Rouch JC, Espenan JM, Clifton M, et al. (2002) From ultrafiltration to nanofiltration hollow fiber membranes: a continuous UVphotografting process. Desalination 144: 9-14.

10. Mbareck C, Nguyen QT, Alexandre S, Zimmerlin I (2006) Fabrication of ion -exchange ultrafiltration membranes for water treatment. I. Semiinterpenetrating polymer networks of polysulfone and poly (acrylic acid). J Memb Sci 278: 10-18.

11. Mbareck C, Nguyen QT, Alaoui OT, Barillier D (2009) Elaboration, characterization and application of polysulfone and poly acrylic acid blends as ultrafiltration membranes for removal of some heavy metals from water. $\mathrm{J}$ Hazard Mater 171: 93-101.

12. Polymer handbooks (1999) Brandrup pages VII/701, 705, 711.

13. Guan Y, Jiang W, Zhang W, Wan G, Peng Y (2001) Polytetrahydrofuran amphiphilic networks. IV. Swelling behaviour of poly (acrylic acid)-Ipolytetrahydrofuran and poly(methacrylic acid)-l-polytetrahydrofuran networks, J Polym Sci A- Polym Chem 39: 1784-1790.

14. Ruaan RC, Chang T, Wang DM (1999) Selection criteria for solvent and coagulation medium in view of macrovoid formation in the wet phase inversion process. J Polym Sci: Part B: Polymer Physics 37: 1495-1502.

15. Albrecht W, Weigel Th, Tiedemann MS, Kneifel K, Peinemann PK, et al. (2001) Formation of hollow fiber membranes from poly(ether imide) at wet phase inversion using binary mixtures of solvents for the preparation of the dope. $J$ Membr Sci 192: 217-230. 
Citation: Mbareck C, Nguyen QT (2014) Study of Polysulfone and Polyacrylic Acid (PSF/PAA) Membranes Morphology by Kinetic Method and Scanning Electronic Microscopy. J Membra Sci Technol 5: 132. doi:10.4172/2155-9589.1000132

16. Smolders CA, Reuvers AJ, Boom RM (1992) Microstructures in phase-inversion membranes Part 1. Formation of macrovoids. J Memb Sci 73: 259-275.

17. Reuvers AJ, Van der Berg JWA, Smolders CA (1987) Formation of membranes by means of immersion precipitation. I. A model to discribe mass transfer during immersion precipitation. J Memb Sci 34: 45-67.

18. Ismail AF, Lai PY (2003) Effects of phase inversion and rheological factors on formation of defect-free and ultrathin-skinned asymmetric polysulfone membranes for gas separation. Separ Purif Tech 33: 127-143.

19. Pekny MR, Greenberg AR, Khare V, Zartman J, Krantz WB, et al. (2002) Macrovoid pore formation in dry-cast cellulose acetate membranes: buoyancy studies. J Membr Sci 205: 11-21.

20. Machado PST, Habert AC, Borges CP (1999) Membrane formation mechanism based on precipitation kinetics and membrane morphology: at and hollow fiber polysulfone membranes. J Memb Sci 155: 171-183.

21. Mckelvey SA, Koros WJ (1996) Phase separation, vitrification, and the manifestation of macrovoids in polymeric asymmetric membranes. J Membr Sci 112: 29-39.
22. Han MJ, Bhattacharyya D (1995) Changes in morphology and transport characteristics of polysulfone membranes prepared by different demixing conditions. J Membr Sci 98: 191-200.

23. Wu L, Sun J, Wang Q (2006) Poly (vinylidene fluoride)/polyethersulfone blend membranes: Effects of solvent sort, polyethersulfone and polyvinylpyrrolidone concentration on their properties and morphology. J Membr Sci 285: 290-298.

24. Tanaka H (1999) Viscoelastic model of phase separation in colloida suspensions and emulsions. Phys Rev E 59: 6842-6852.

25. Kim JH, Lee KH (1998) Effect of PEG additive on membrane formation by phase inversion. J Memb Sci 138: 153-163.

26. Han MJ, Nam ST (2002) Thermodynamic and rheological variation in polysulfone solution by PVP and its effect in the preparation of phase inversion membrane. J Memb Sci 202: 55-61. 